\title{
What makes an effective energy efficiency label? Assessing the performance of energy labels through eye-tracking experiments in Ireland
}

\begin{abstract}
Energy labels are a means of providing consumers with a way of understanding the environmental and energy impacts associated with many products and services. These labels are designed to help consumers to compare options and to select more energy efficient products. Such labels often contain large amounts of information, displayed in a range of metrics, such as carbon dioxide emissions in grams, energy use in kilowatt hours, and noise emissions in decibels. This paper presents the results of an eye-tracking experiment undertaken to assess energy labels from both the whitegoods and motor sectors. Specific focus is put upon the elements that make up these labels, and how effective these elements are at transmitting the necessary information to the consumer. Based upon the results of this study it is possible to generate heat maps, scan paths, and statistical analysis formed from defined areas of interest within the energy labels. These results highlight the effectiveness of colour coding (traffic light style) and simple alphabetic grading, in terms of drawing the attention of consumers. This research also contained information recall tasks based upon the data provided to respondents, and an analysis of these results highlights the notable variation in recollection accuracy for the different types of information provided by the labels. Based upon these results, a number of recommendations are made concerning potential label design.
\end{abstract}

\section{Introduction}

It has long been seen that one of the solutions to reducing energy usage and promoting efficiency will be via behavioral change and social scienc has a key role to play (Sovacool, 2014) Energy labels are a graphical means of provided consumers with information about the environmental and energy impacts associated with a number of product and services. These labels come in a wide range of formats, from simple logos to much more complex labels. Complex labels, such as those mandated across a number of industries in the European Union and neighbouring nations, often provide large amounts of information in the form of different metrics. These may include energy efficiency ratings within a given class, estimated annual energy use in kilowatt hours, associated noise in decibels, emissions in grams per kilometre of driving, fuel use in different driving regimes and environments, and annual water consumption estimates. In addition to the complexity of these labels, it must be considered that any information provided by such a label is unlikely to be processed in isolation, rather it will be considered along with other product attributes such as brand, price, and warranty. In the design of energy labels it is clear that designers have given more prominence to certain factors over others, by making decisions regarding features such as text size and colour, position on the label, and use of imagery. Given the diversity of styles utilised within such labels, it is unlikely that all information will received the same level of attention.

\section{Literature Review}

Behavioral changes to promote energy efficiency has been examined in a number of different ways in the literature. A number of studies have used innovative methods to promote this change. Timm and Deal (2016) used a public real-time energy consumption dashboard at a University campus to promote awareness and found this reduced energy and gas consumption. Smart phone applications have also been used to provide information to individuals to change behaviors (van der Werff and Steg, 2016). Information from smart metering has also been shown to be an effective way to reduce 
energy consumption ( $D^{\prime} \mathrm{O}$ ca et al, 2014). Our study falls into this field of using technology and information to determine how energy reductions can be achieved.

\subsection{Energy Labels}

With changes in the global climate and the reduction in availability of easily accessed fossil fuels, there is an increasing need to reduce the amount of energy expended by devices that are used as part of everyday living. This will require an informed population making decisions to limit their impacts on the environment but empowering individuals to make these decisions can be difficult. One means of addressing this concern is the provision of energy labels and ratings, which in theory have the ability to enable consumers to make more sustainable choices, by increasing their awareness of the impacts of their choices, in terms of energy use and therefore running associated running costs. Much of the information that is displayed by such labels would otherwise be unavailable to consumers, and therefore their ability to make sustainable choices would be severely hampered if such labels were not provided. The provision of such information is a legal requirement in a number of sectors in the European Union, including white goods, housing, the motor industry, and electronic.

Within the European Union, the provision of energy labels is required by Directive 2010/30/EU. This legislation also outlines the reasoning behind the requirement to supply such labels, stating: "The provision of accurate, relevant and comparable information on the specific energy consumption of energy-related products should influence the end-user's choice in favour of those products which consume or indirectly result in consuming less energy and other essential resources during use, thus prompting manufacturers to take steps to reduce the consumption of energy and other essential resources of the products which they manufacture" (EU, 2010). Based upon this legislation, it is apparent that such labels are designed with multiple, though related, purposes. Namely these are the provision of information to allow consumers to make comparisons between goods, with the intention of promoting a shift towards items that consume less resources and energy. In addition to this, the provision of energy labels is viewed as means of motivating manufacturers to product more energy efficient goods, under the assumption that the goods with higher ratings will become more competitive within the marketplace. For such measures to work it is therefore important that consumers are able to understand and easily use the information that the labels provide.

\subsection{Energy Information Presentation}

Energy labels provide information that consumers would not otherwise encounter, and therefore in many cases will be unfamiliar with these information. Such labels often provide additional visual and comparative aids to help consumers process the information. In the case of energy labels two methods that are often employed are the use of alphabetical rating scales with appliances being given ratings such as $\mathrm{A}$ or $\mathrm{B}$, and the use of colour coded scales, where green generally denotes higher levels of energy efficiency and red lower levels. This type of scaling with colour coding has been shown to be effective in changing customer preferences (Waechter et al, 2016). This colour coding approach is used in sectors ranging from the airline sector (Flybe, 2017) to the construction and glazing industries (NSAI, 2017). Similar methods are also employed in the housing sector where Building Energy Ratings certificates provide individuals with information regarding the energy efficiency of a dwelling or commercial space (SEAl, 2017). However, while research has shown that there such information may have a value to consumers in a number of sectors (Shen and Saijo, 2009; Gaspar and Antunes, 2011; Sammer and Wustenhagen, 2006), research has also suggested that consumers still do not have a good understanding of information associated with sustainability (Whitmarsh et al, 2011). Even with regard to the current design of energy labels in the white goods 
sector, concerns have been raised regarding whether such labels are as effective as they could be, specifically in terms of providing consumers with meaningful comparisons between products (Heinzle and Wustenhagen, 2012).

\subsection{Eye-tracking Applications}

Eye-tracking is a method whereby light is reflected of the pupils of a participant looking at a stimulus, and based upon the recordings of these reflections, it is possible to track the movement of their gaze. Using these tracks researchers can then determine what information the respondents have looked at, and what information they may have ignored. While attention to a given area of an image or label does not necessarily mean that the individual considered such information to be of importance, non-attendance to information would suggest that such information is not of value, and will not be considered when making choices. From the perspective of energy labels, the application of eye-tracking has the potential to assess which elements of a label attract consumers' attention, which elements they focus upon, and how they interact with the information provided. Eye tracking methods have been used in many different fields. For example, Linert et al (2017) use it to measure the acceptance of high voltage cables, Wolfson et al (2017) use it to examine how we examine calorie information and Dzeng et al (2016) use it for hazard identification on construction sites. This study uses the technology to measure how we examine eco-labels.

\subsection{Eye-Tracking and Label Design}

Eye-tracking has been used in a variety of sectors to gain more information about how individuals acquire and understand information. There are a number of studies in the marketing industry that have utilised this technology, specifically examining the roles of labels and label elements.

One area that had received specific interest, in terms of the application of eye-tracking to assess label effectiveness, is with regard to the provision of nutritional information on food products. Very similarly to energy information, nutritional information can be linked to long term benefits for consumers if properly utilised, however it also must compete with other information for consumer attention while being provided in units that consumers may not have a large degree of experience with. Wolfson et al (2017) used an eye-tracker to look at the presentation and contextualisation of calorie information, in terms of physical activity equivalents, and found this approach to be more effective than numerical information. Similarly, research into "traffic light" contextualisation approaches, similar to those used in energy labels, utilised eye-tracking to assess nutritional information and traffic light contextualisation, where traffic light formats were found to take less time to process than the numerical guideline daily amounts (Siegrist et al, 2015). While this would appear to reflect positively on such an approach research by Mawad et al (2015) found that the use of such formats can be modulated by the consumers' cognitive styles.

In one of the very few studies looking at the role of energy labels, in this case in both the white goods and television markets in Switzerland, Waechter et al (2015) utilised eye-tracking technology to assess the levels of attention paid to various specifications and information. The results of their research highlighted the role that eye-tracking can play in assessing energy information, and helped establish a new research field that this research builds upon.

\section{Methodology}

The application of eye-tracking technology to eco-labels was viewed as providing the research team with a unique understand of how such information is attended to and assessed by consumers. For this study a sample of 43 individuals was recruited primarily from the university population in late 
2016. While the sample collected could be considered small the results presented demonstrate how the technology can be used in this field of evaluation.

\subsection{Sample}

Table 1 presents a description of the sample used for the purposes of this research in terms of the age and gender split of the participants. As the research was carried out largely in a university setting, the group tends to be younger and better educated than the population as a whole, with more males than females.

Table 1: Sample Properties

\begin{tabular}{|cc|}
\hline Age & Standard Deviation \\
29.5 & 9.96 Years \\
\hline Male & Female \\
26 & 17 \\
\hline
\end{tabular}

\subsection{Experimental Set Up}

For the purposes of this experiment participants were asked to take part in a half an hour session to explore the role of eco-labels in terms of informing consumer choices. Participants sat at a desk approximately $70 \mathrm{~cm}$ away from a computer screen below which the eye-tracker was placed (see Figure 1). This screen then presented a number of images relating to energy labels for both white goods and car sales. The eye-tracker operates as the bar emits beams of near infrared light which are then reflected off participants' pupils, the position of these reflections is recorded, and using the accompanying software it was able to map their gaze across the screen. These gaze traces could then be mapped onto the stimuli to assess what elements of the labels participants were attending to. Figure 1 provides an outline of the experimental set up.

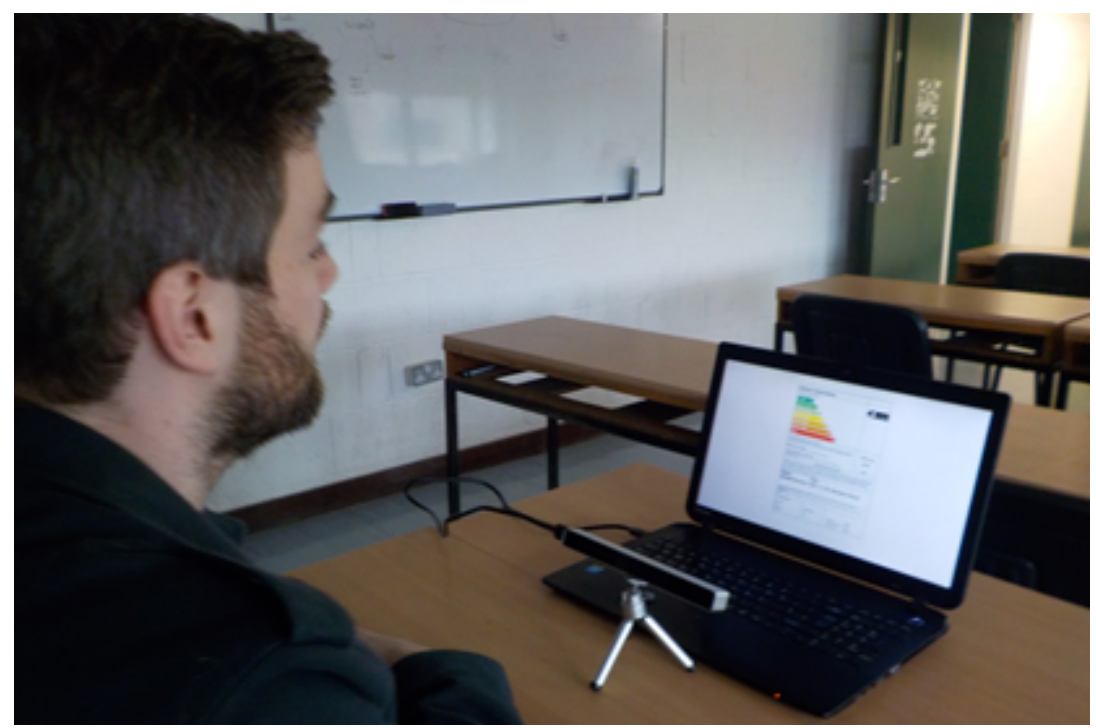

Figure 1: Experimental Set Up

Before taking part in the eye-tracking segment of the experiment, participants were asked to take part in a number of calibration tasks, both to ensure that they were comfortable with the experimental setup, and also that the equipment was operating correctly. For the purposes of the experiment, study participants were shown two images of typical energy labels, one from the white goods sector and one from the motor sector, and then they were asked to look at the labels and 
take in the information provided. These images were selected as they contained a number of elements commonly observed in energy labels, including colour coded rating scales with attached alphabetical values, as well as more detailed information that tends to be specific to the industry or sector in which the label occurs. These images are provided in the results section to allow ease of comparison with the heat maps generated by participants.

The experiment placed no time limit was placed upon participants, and they could exit the program once they were happy they understood the information provided. This was undertaken with the intention of mimicking browsing information that may occur either in a showroom or retail setting, or while the individual is researching a product online. Once participants had viewed the images and were happy that they had taken in all the information they required, they were asked a number of questions relating to the information provided by the label. Specifically, these questions required participants to attempt recall the information displayed in both of the labels shown, including the energy ratings, the colour code associated with the ratings, associated emissions, fuel, and electricity consumption. This study was conducted following the receipt of ethical approval. Figure 2 outlines the steps that constituted the experiment.

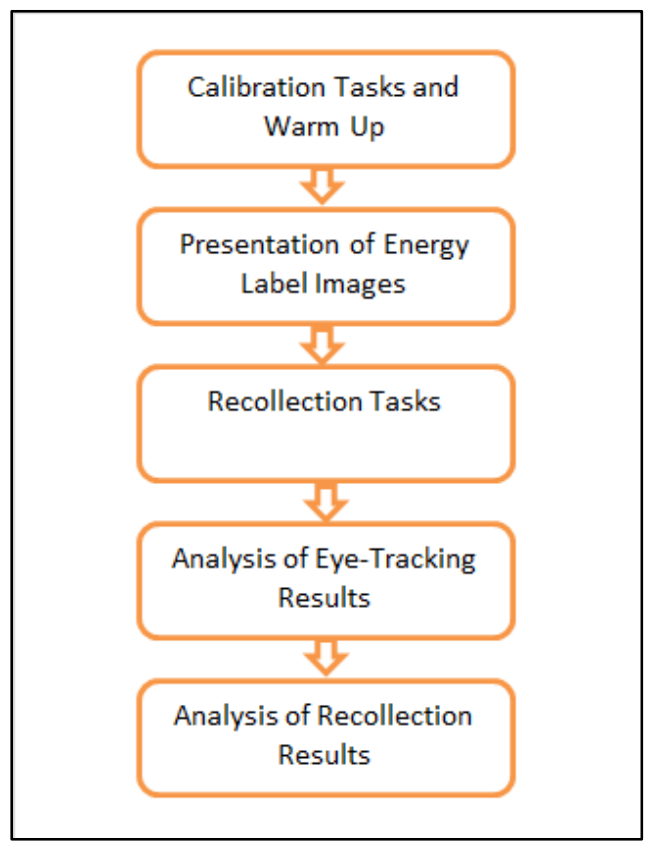

Figure 2: Experimental Process

\section{Results}

For the purposes of this study, a number of analysis techniques were used to enable a holistic examination of the research area. One of the advantages of eye-tracking technology is its ability to produce a number of outputs to allow analysts to understand the participants' interactions with the stimuli. Within this field one of the most commonly utilised methods of examining the underlying data generated from the participants' tracks is the use of heat maps.

\subsection{Heat Maps}

Heat maps provide the analyst with the most intuitive means of examining the data generated from the observations, and therefore makes a good starting point to understand the results. These maps are generated based upon the duration that respondents fixated upon a given section of the image, with brighter more intense colours indicating areas with increased levels of fixation. 
Figure 3 outlines the results for the label associated with the motor industry. This map is constructed based upon relative fixation duration, that is the percentage of time spent looking at the image that is focused upon a given section. An initial examination of these images shows that there are a number of hot spots scattered across the image, and these correspond to prominent information in the original image. The most pronounced of these spots are located on top of the colour coded rating followed by the information relating to the running cost of the car and its annual tax and VRT (Vehicle Registration Tax) rate. Other centres of attention correspond to the fuel consumption information at the bottom of the image and the details of the make and model of the car.
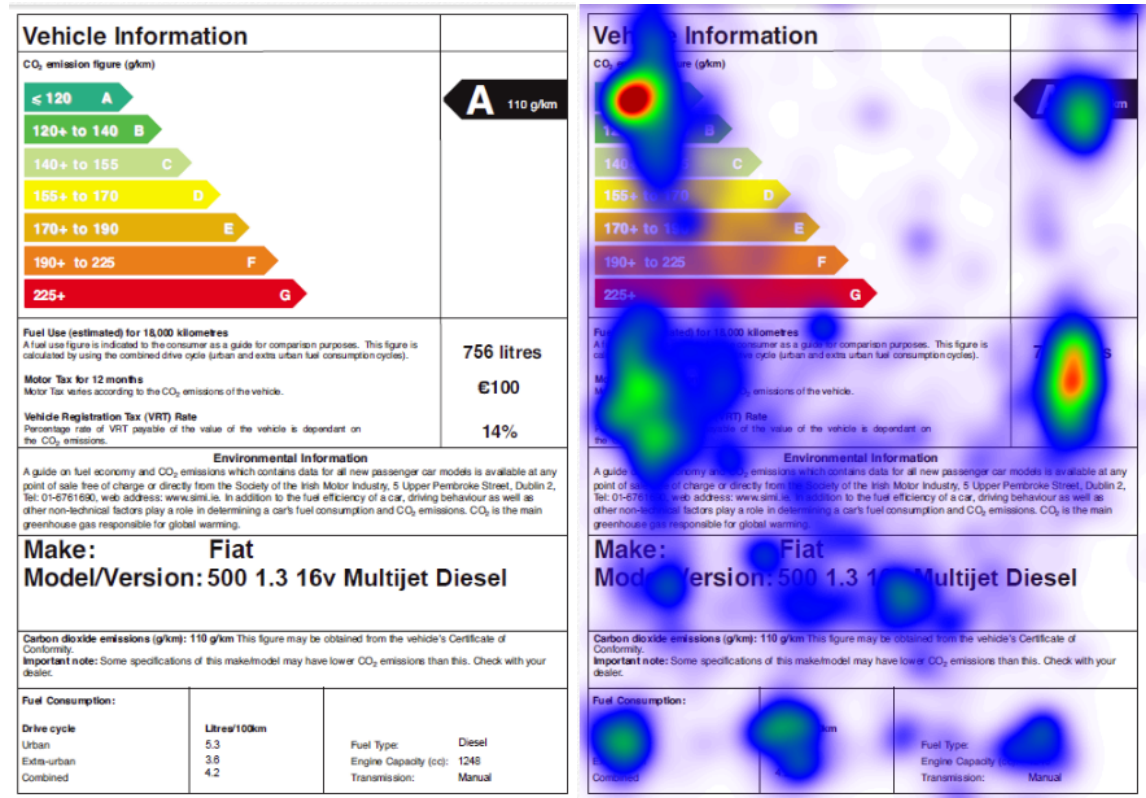

Figure 3: Car Label and Heat Map

An examination of the heat map image relating to the white goods sector provided by Figure 4 displays a similar pattern to that seen for the car label, although there is less information being presented in this image. In the case of this label it can be seen that there is a concentration of attention located over the energy ratings and colour coded grading scheme. This once again suggests that the letter ratings provided in conjunction with the colour coded scale are the element of the label that consumers are most drawn to. As with the car label, it can be seen that only the section of the colour coded scale that corresponds to the value associated with the appliance is highly attended, and that the lower ratings and red section of the scale are largely ignored. 


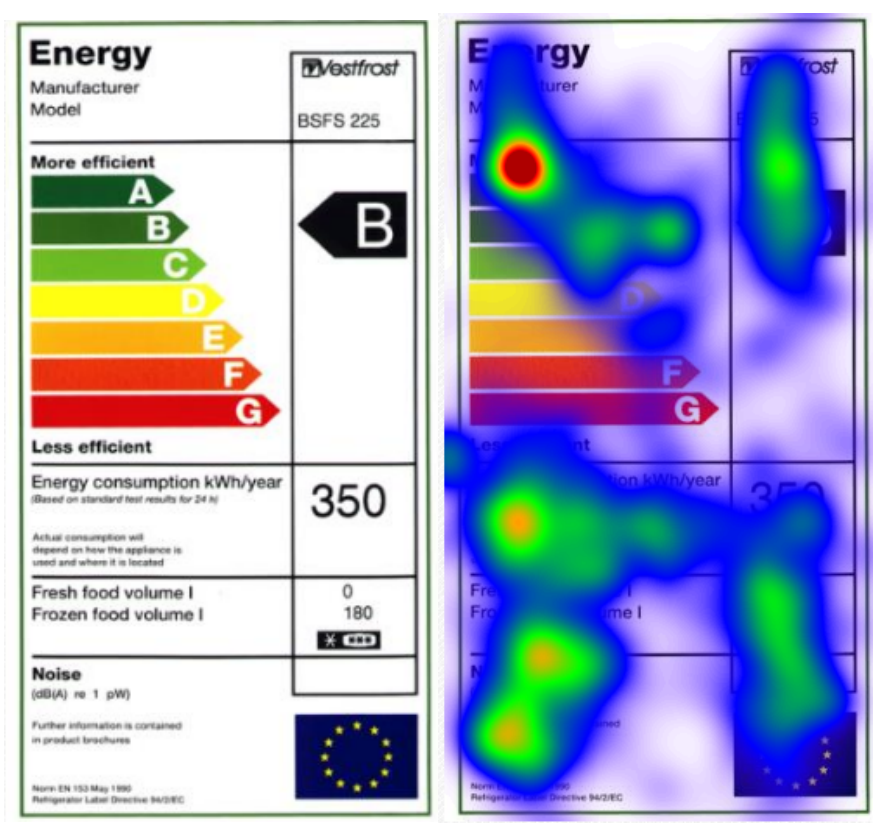

Figure 4: White Goods Label and Heat Map

\subsection{Areas of Interest}

For each of the images there were a number of areas of interest (AOIs) defined by the research team. These AOls are provided for the car label and the white goods label in Figures 5 and 6 respectively. Figure 5 outlines the AOls defined for the motor sector label, including: Colours, Ratings, Fuel Use and Tax, Cost, Environmental information, Make and Model, Carbon Dioxide Information, and Fuel Consumption. These AOls were designed to be non-overlapping and to capture the principal components of the label. 


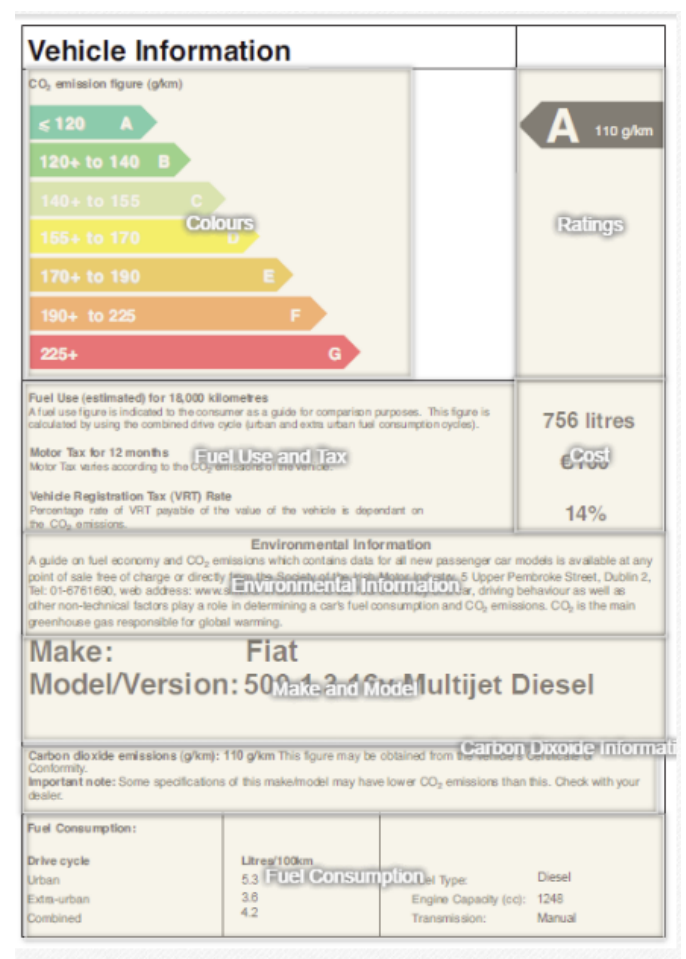

Figure 5: Car Label AOIs

Table 2 displays the initial statistics for the AOls. This table displays both the number of fixations, which is the mean number of times participants fixated on information in a given $\mathrm{AOI}$, and the time to first fixation, which is the average amount of time from when the participant first viewed the image to when the first focused their gaze upon a given AOI. These metrics provide information on which elements of the label the participants focused on the most, and which elements first captured their attention. The results displayed in Table 2 show a pattern of first fixation progressing vertically down the image, with the colour coded scale and the emissions rating are the first elements to be fixated upon. It is notable that the colour scale is the first aspect of the label to be fixated upon, as this approach is often the only information provided in more simplified labels. An examination of the number of fixations in each $\mathrm{AOI}$ shows much higher levels of attendance on the colour coded scale, the make and model of the vehicle, and the most notably the fuel use and tax information associated with the car. The fuel use and tax rates associated with the vehicle have a direct link to running costs, and therefore there may be a financial motivation to investigate this information.

Table 2: Car Label Statistics

\begin{tabular}{|l|c|c|}
\hline & Time to first fixation & Number of fixations \\
\hline Rating (Letter) & $2,036 \mathrm{~ms}$ & 5.64 \\
\hline Colours & $1,129 \mathrm{~ms}$ & 10.74 \\
\hline Fuel Use and Tax & $5,658 \mathrm{~ms}$ & 13.44 \\
\hline Cost & $6,588 \mathrm{~ms}$ & 6.08 \\
\hline Environmental information & $11,680 \mathrm{~ms}$ & 6.08 \\
\hline Make and Model & $15,474 \mathrm{~ms}$ & 10.58 \\
\hline
\end{tabular}




\begin{tabular}{|l|c|c|}
\hline Carbon Dioxide Information & $30,072 \mathrm{~ms}$ & 9.81 \\
\hline Fuel Consumption & $28,124 \mathrm{~ms}$ & 4.19 \\
\hline
\end{tabular}

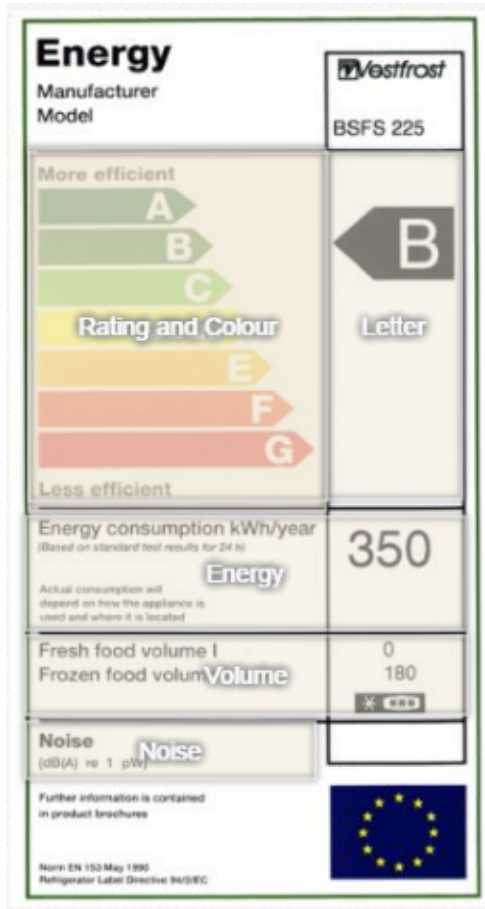

Figure 6: White Goods Label AOls

Table 3 outlines the results arising from the AOls associated with the white goods label presented in Figure 6. An examination of the time to first fixation shows that, as with the car label, the colour coded scale in the top left corner of the label is the first element of the label to attract participants' attention. This is followed by the energy rating in the top right section of the image and the energy information in the centre of the image. Both volume and noise information are on average fixated on at a much later stage than the other label elements. When analysing the number of fixations each $\mathrm{AOI}$ received, it is clear that participants fixated more times on the energy information in the upper section of the image, with values nearly double those observed for the appliance volume information and triple that observed for the noise information. Again, these results demonstrate the appeal of the colour coded scales, in terms of attracting consumers' attention.

Table 3: White Goods Label Statistics

\begin{tabular}{|l|c|c|}
\hline & Time to first fixation & Number of fixations \\
\hline Rating (Letter) & $2715 \mathrm{~ms}$ & 10.5 \\
\hline Colour & $154 \mathrm{~ms}$ & 10.92 \\
\hline Energy & $2706 \mathrm{~ms}$ & 11.7 \\
\hline Volume & $5889 \mathrm{~ms}$ & 5.73 \\
\hline Noise & $9170 \mathrm{~ms}$ & 3.56 \\
\hline
\end{tabular}




\subsection{Recollection Tasks}

The final metric used to test the effectiveness of the labels was a recollection task. The purpose of this task was to assess which elements of the label had been the most effective in terms of informing the participants. This was designed to complement the information recorded by the heat maps and AOIs, and to provide some more context to the data they display. Two sets of recollection tasks were created, one associated with each of the labels. These tasks took place once the participant had seen both images, so as not to prepare them and to hopefully imitate a more natural observation state. While the ability of participants to recall information may be influenced by nature of the experiment, as the primary focus of research was the effectiveness of the means of communication relative to each other, it was felt that this would not present a major concern.

\subsubsection{Car Recollection Task}

In the case of the car label information recollection task, nine values were considered for analysis. An assessment of the results presented in Table 4 shows that the letter rating, in this case $A$, was the piece of information that was accurately recalled by the largest number of participants, with $90 \%$ correctly identifying it. Apart from this, only information regarding the make of the car and the associated motor tax were recalled accurately by the majority of participants. From an environmental perspective only $36.5 \%$ of respondents were able to recall the estimated emissions associated with the vehicle to within $10 \%$ of the correct value. Perhaps the most striking results is the proportion of participants who were able to recall the fuel use associated with the three driving styles outlined by the label, where in each case the vast majority of participants were unable to accurately recall these values. The results arising from this section of the experiment outline the importance of providing easily understood and quickly comparable metrics, such as the alphabetical grades, over more specialised information. These results also demonstrate the wide disparity, in terms of information retention, that exists between the various metrics displayed by energy labels. While the recollection tasks do not map one to one onto the eye-tracking AOls due to label design, it is interesting to note the wide disparities in recollections between information that is presented together, such as the letter rating and carbon dioxide emissions estimates.

Table 4: Car Label Recollection Task

\begin{tabular}{|l|c|c|}
\hline & Correct Value & \% Correct (+/- 10\%) \\
\hline Letter Rating & $\mathrm{A}$ & $90 \%$ \\
\hline Emissions & $110 \mathrm{~g} / \mathrm{km}$ & $36.5 \%$ \\
\hline Fuel Use & 756 & $39 \%$ \\
\hline Motor Tax & $€ 100$ & 68.3 \\
\hline VRT & $14 \%$ & $34.9 \%$ \\
\hline Make and Model & Fiat & $67.4 \%$ \\
\hline Urban Fuel/100km & 5.3 & $9.3 \%$ \\
\hline Extra-Urban & 3.6 & $4.6 \%$ \\
\hline Combined & 4.2 & $6.9 \%$ \\
\hline
\end{tabular}

\subsubsection{White Goods Recollection Task}

The second recollection task related to the white goods label and can be considered to be the simpler of the two labels under consideration, as it contained only three pieces of information. These areas and their associated scores are outlined in Table 5 in terms of the percentage of study 
participants who could correctly remember their values. In the case of numerical values, if participants provided figures that were within $10 \%$ of the value displayed on the label, these answers were accepted as being correct. It can be seen that, in terms of communicating information regarding the energy consumption of the appliance, the alphabetical rating scale was the most effective, with $76 \%$ of participants correctly recalling that the appliance had a B grade. $60 \%$ of participants remembered that the colour green had been associated with the appliance, while only $30 \%$ of respondents were able to recall that the unit had an energy consumption of 350 kilowatt hours per year. Even within a relatively simple label such as this, with only a few pieces of information being provided, it is clear that there is considerable variation in the ability of participants to recall what they have read.

Table 5: White Goods Recollection Task

\begin{tabular}{|l|c|c|}
\hline Question & Correct Value & Correct (+/- 10\%) \\
\hline What was the energy rating (A-G) & B & $76 \%$ \\
\hline What was the energy consumption (Numerical) & $350 \mathrm{kwh} /$ year & $30 \%$ \\
\hline What was the colour code for this product & Green & $60 \%$ \\
\hline
\end{tabular}

\section{Discussions}

The experiments outlined in this paper were designed to provide a better understanding of the effectiveness of various methods of transmitting information via energy labels. These labels are designed to provide consumers with information that might otherwise be unavailable to them, however this information is not presented in a uniform manner across the labels, rather certain product attributes are highlighted via the use of large bold fonts and the use of a scaled colour scheme. The effectiveness of these methods can be clearly be seen in the results presented in the previous section. For both of the labels used in this experiment, respondents were initially drawn to the information provided in the top left hand corner of the image. In the case of the motor sector label this information relates to carbon dioxide emissions, whereas for the white goods image it related to the energy efficiency of the appliance.

The results of the recollection tasks confirmed the differences in the effectiveness of the various information presentation methods, in terms of their ability to transfer information to the consumer which can be called upon at a later time. For both labels, the alphabetic rating provided in the upper left corner of the image was the information successfully recalled by the largest number of study participants. Large amounts of information provided by both labels could not be recalled by the majority pf participants, suggesting that as such information is not easily remembered, it can only play a very limited role in terms of informing the consumers choice. It should also be noted that this information displayed low recall rates in an experimental setting where no other information was on display, whereas in a retail environment information regarding product attributes such as price or features would also be competing for the consumer's attention.

The effectiveness of the alphabetical rating approach is especially clear for the label in the car industry, as while this element of the label received significantly less fixations than other parts of the label, it was recalled by a large majority of participants. This highlights the ability of such an approach to be viewed quickly, easily understood, and then recalled later potentially for comparative purposes. 


\section{Conclusions}

Energy labels are designed and employed as a means of providing consumers with information regarding the environmental and energy impacts that might not already be available to them otherwise. Such labels may employ various graphical methods to highlight such information, however, when labels are attempting to transmit complex information, or even a number of complex metrics all at one time, designers must decide which aspects of the good are afforded prominence at the expense of others. The results of this study, both those associated with the eyetracking element of the research and those from the information recollection tasks, demonstrate that the attention participants give to various elements within a label can vary considerably, and that the ability of label elements to provide information that can easily be recalled can vary considerably.

If consumers are only considering simple easily remembered information such as alphabetical grades or colours when assessing, there is an onus on label designers to ensure that such scales encompass all the information they deem to be important to promote consumption in line with policy objectives, such as reduced energy and fuel use or reduced greenhouse gas emissions. The results of this research would also appear to indicate that large amounts of information are being discarded by consumers, however such information, such as carbon dioxide emissions estimates, is often complex to calculate and requires the expenditure of resources to provide and thought may be needed as to how to better communicate it. When it is considered that energy information is only one factor in a consumers' decision when making a purchase, it is important that they are receiving the appropriate data.

In more general terms energy labels have the ability to provide consumers with information regarding energy consumption and environmental impacts that would otherwise be unavailable to them. If these labels are designed in such a manner that there is a consistency of approach across a number of sectors, such as is seen with colour coded alphabetical scales, there is greater potential for such labels to play a more prominent role in consumer choices.

\section{Acknowledgements}

This research was funded by the Environmental Protection Agency (Ireland) under their sustainability research program.

\section{References}

D'Oca, S., Corgnati, S.P., Buso, T. Smart meters and energy saving in Italy: Determining the effectiveness of persuasive communication in dwellings. Energy Research \& Social Science, Volume 3, September 2014, Pages 131-142

Dzeng, R., Lin, C., Fang, Y. Using eye-tracker to compare search patterns between experienced and novice workers for site hazard identification. Safety Science, Volume 82, February 2016, Pages 56-67

FLYBE. 2016. Ecolabelling Scheme. Accessed 28.02.2017. Available at: http://www.flybe.com/corporate/sustainability/eco_labelling_scheme.htm

Heinzle and Wustenhagen. 2012. Dynamic Adjustment of Eco-labeling Schemes and Consumer Choice-the Revision of the EU Energy Label as a Missed Opportunity. Business Strategy and the Environment, 21, 60-70 
Gaspar, R., and Antunes, D. 2011. Energy efficiency and appliance purchases in Europe: Consumer profiles and choice determinants. Energy Policy, Vol. 39, 7335-7346

Lienert, P., Sütterlin, B., Siegrist, M. The influence of high-voltage power lines on the feelings evoked by different Swiss surroundings. Energy Research \& Social Science, Volume 23, January 2017, Pages 46-59

Mawad, F., Trias, M., Gimenez, A., Maiche, A., and Ares, G. 2015 Influence of cognitive style on information processing and selection of yogurt labels: Insights from an eye-tracking study. Food Research International, Vol. 74, p1-9.

National Standards Authority Ireland. 2017. Window Energy Performance Scheme. Accessed 28.02.2017. Available at http://www.nsai.ie/Our-Services/Certification/Agrement-Certification/WEP(Wind-Energy-Performance)-Scheme.aspx

Oliveria, D., Machin, L., Deliza, R., Rosenthal, A., Walter, E.H., Gimenez, A., and Ares. G. 2016. Consumers' attention to functional food labels: Insights from eye-tracking and change detection in a case study with probiotic milk. LWT-Food Science and Technology, Vol. 68, p160-167.

Sammer. K. and Wustenhagen, R. 2006. The Influence of Eco-Labelling on Consumer BehaviourResults of a Discrete Choice Analysis for Washing Machines. Business Strategy and the Environment, $15,185-199$

Shen, J., and Saijo, T. 2009. Does an energy efficiency label alter consumers' purchasing decisions? A latent class approach based on a stated choice experiment in Shanghai. Journal of Environmental Management, 3561-3573

Sovacool, B.K. Diversity: energy studies need social science, Nature 511 (7511) (2014) 529-530

Siegrist, M., Leins-Hess, R., and Keller, C. 2015. Which front-of-pack nutrition label is the most effective one? The results of an eye-tracker study. Food Quality and Preference, Vol. 39, p 183-190

Sustainable Energy Authority Ireland (SEAI). 2017. BER FAQ. Accessed 28.02.2017. Available at: http://www.seai.ie/Your_Building/BER/BER_FAQ/FAQ_BER/General/What-is-a-BER-.html

Timm, S.N., Deal, M.B. Brian M. Effective or ephemeral? The role of energy information dashboards in changing occupant energy behaviors. Energy Research \& Social Science, Volume 19, September 2016, Pages 11-20

van der Werff, E., Steg, L. The psychology of participation and interest in smart energy systems: Comparing the value-belief-norm theory and the value-identity-personal norm model. Energy Research \& Social Science, Volume 22, December 2016, Pages 107-114

Waechter, S., Sutterlin,B., and Siegrist, M. 2015. Desired and Undesired Effects of Energy Labels-An Eye-Tracking Study. PLoS One 10(7)

Waechter, S., Sütterlin, B., Borghoff, J., Siegrist, M. Letters, signs and colors: How the display of energy-efficiency information influences consumer assessment of products. Energy Research \& Social Science, Volume 15, May 2016, Pages 86-95

Wolfson, J.A., Graham, D.J., Bleich, S.N. Attention to physical activity-equivalent calorie information on nutrition facts labels: An eye-tracking investigation. Journal of Nutrition Education and Behavior, Volume 49, Issue 1, January 2017, Pages 35-42 
Whitmarsh, L., G, Seyfang, and O'Neill,S. 2011. Public engagement with carbon and climate change: To what extent is the public 'carbon capable'? Global Environmental Change, 21, pp 56-65.

Wolfson, J.A., Graham, D.J., Bleich, S.N. 2017. Attention to Physical Activity-Equivalent Calorie Information on Nutrition Facts Labels: An Eye-Tracking Investigation. Journal of Nutrition Education and Behaviour, Vol. 49, Issue 1, pp 35-42. 